\title{
QSAR Models for Cytotoxicity of Chlorinated Alkanes
}

\author{
R.Suganya, S.Bharanidharan, R.Velavan
}

\begin{abstract}
Knowledge on the degree of toxicity of aliphatic hydrocarbons and their derivatives has become essential for environmental safety and security. In the present study, an effort has been put forth to analyze the toxicity of aliphatic hydrocarbons. This work focuses on development of new QSAR models with various DFT based descriptors. Cytotoxicity of Chlorinated alkanes with experimental activity log EC50 Values are utilized to obtain QSTR models.
\end{abstract}

Key words: QSAR/ QSTR, DFT electrophilicity index $(\omega)$, polarizability $(\alpha)$, chemical potential $(\mu)$, and hardness $(\eta)$

\section{INTRODUCTION}

Quantitative Structure Activity Relationship (QSAR) and Quantitative Structure Toxicity Relationship (QSTR) have seen recent upsurge of interest because of their ability in predicting various activities and properties of complex molecules. QSAR is a powerful research tool to successfully establish / predict activity models and evaluate the toxicity of those chemicals introduced or not introduced into the markets. Such theoretical evaluation of toxicity of chemicals reduces the experimental efforts in identifying its effect on living forms. [8],[10] ,[12]

A QSAR model has to satisfy a number of conditions related to the different aspects of the model (Wold et al 1983, Eriksson et al 2001). The biological activity/toxicity of a series of compounds should be related to the physico-chemical properties being considered. Also, the activities of the compounds should be elicited by common and relevant mechanism. Related chemical structures are not strictly required but structures with unknown mechanism of action are not encouraged. Hence, chemically similar compounds with same mechanism of action are used for generating QSAR models. [7],[ 9],[11]

A mathematical formulation on "the principle for toxicity" was first coined by Ferguson (1939). It has been observed that the toxicity of aliphatic and aromatic hydrocarbons to mammals is extremely variable, depending on the species, compound, and environmental conditions (UNEP 2006). Alkanes are a class of aliphatic hydrocarbons having very low (aquatic) toxicity. The introduction of chlorine atoms into the hydrocarbon chain alters properties such as

Revised Manuscript Received on July 22, 2019.

R. Suganya, Department of physics, Bharath Institute of Higher education and research, Chennai , India Email: suganya_s_r@ yahoo.com

Dr. S.Bharanidharan Department of physics, Bharath Institute of Higher education and research, Chennai , India Email: bharani.dharan0@gmail.com

Dr.R.Velavan Department of physics, Bharath Institute of Higher education and research, Chennai , India.Email: prvelavan@gmail.com solubility, density, volatility, and toxicity (NRC 1993). Several studies have highlighted the acute toxicity of chlorinated alkanes.

Quantum chemical descriptors based on density functional theory (DFT) (Parr \& Yang 1989; Chattaraj 2009; Chattaraj \& Giri 2009) have become quite successful in explaining physicochemical properties (Giri et al 2008) and toxicity (Roy et al 2006, Padmanabhan et al 2006) of diverse classes of chemical compounds and drug molecules. [1],[3],[5]

\section{COMPUTATIONAL DETAILS}

The three-dimensional conformation of the compounds and their derivatives are built using Cerius2 package and are optimized using the semi empirical quantum chemical method, Austin Method 1 (AM1) to obtain the stable conformation. The conformational energy and the atomic / molecular orbital energies (EHOMO and ELUMO) have been obtained by calculating the single point energy at B3LYP/6-31G* level. Using this method, a series of quantum chemical indices, [2 ],[ 4],[6] such as Hartree Fock energy HF (E), the LUMO energy (L), electrophilicity index $(\omega)$, polarizability $(\alpha)$, chemical potential $(\mu)$, and hardness $(\eta)$ have been obtained. Relationship between these parameters has been analyzed to identify whether the activity of these compounds have any influence over these parameters and hence these parameters can be used as a controlling factor for its activity and vice versa. The quantum calculations have been performedusing GAUSSIAN98W package and the structures of studiecompounds has been modeled using Silicon Graphics workstation[13], [15] ,[ 17]

\section{RESULT AND DISCUSSION}

The QSAR analysis on aliphatic and aromatic hydrocarbons have been performed to highlight inhibitor-enzyme interaction mechanisms based on the in silico derived toxicity, which would eventually provide additional information for future molecular design of compounds belonging to this category.

A set of 77 chlorinated alkanes with cytotoxicity activity (log $\mathrm{EC}_{50}$ ) (Zvinavashe et. al.2008) have been selected for this analysis. The studied 
chlorinated alkanes have been classified into two categories based on their hardness values. [32],[34]

Compounds having hardness value greater than 0.15 are listed in Table 1.1a (16 compounds) and those having lesser than 0.15 are listed in Table $1.1 \mathrm{~b}$ (61 compounds). Majority of the compounds in the first set have long hydrocarbon chain (up to ten carbons) with chlorine in the first position. The second set of compounds are shorter in length ( $<10$ carbons) and are more chlorinated ( 3 to 7 chlorines). The parameters such as chemical potential $(\mu)$, electrophilicity index $(\omega)$, dipole moment (DP), polarizability, hydrophobicity $(\log \mathrm{P})$ and relative energy (RE) are used for deriving QSAR equations. [14],[ 16], [18]

Table 1.1a: List of compounds classified as set 1

\begin{tabular}{|c|c|c|}
\hline S.NO & & 1) Compound \\
\hline $\mathrm{A} 1$ & & 1,3-dichloropropane \\
\hline $\mathrm{A} 2$ & & 1,2-dichloropropane \\
\hline A3 & & 1,1,2-trichloroethane \\
\hline A4 & & 1,2-dichlorobutane \\
\hline A5 & & 1-chloro-2,2-dimethylpropane \\
\hline A6 & & 1-chloro-2-methylbutane \\
\hline A7 & & 1-chloropentane \\
\hline A8 & & 1,6-dichlorohexane \\
\hline A9 & & 1-chlorohexane \\
\hline a) & $\begin{array}{l}A 1 \\
0\end{array}$ & 1,1-dichloro-3,3 dimethylbutane \\
\hline A11 & & 1-chloroheptane \\
\hline A12 & & 1,8-dichlorooctane \\
\hline A13 & & 1-chlorooctane \\
\hline A14 & & 1,9-dichlorononane \\
\hline A15 & & 1-chlorononane \\
\hline A16 & & 1-chlorodecane \\
\hline
\end{tabular}

Table 1.1b: List of compounds classified as set 2

\begin{tabular}{|l|l|}
\hline S.NO & Compound \\
\hline B1 & 1, 2, 3-trichloropropane \\
\hline B2 & 1-chlorobutane \\
\hline B3 & 1, 5-dichloropentane \\
\hline B4 & Pentachloroethane \\
\hline B5 & 1, 1, 1, 2, 2, 2, 2, 3-heptachloropropane \\
\hline B6 & Hexachloroethane \\
\hline B7 & 1, 1-dichloroethane \\
\hline B8 & 1, 3-dichloro-2,2-bis(chloromethyl)propane \\
\hline B9 & Trichloromethane \\
\hline B10 & 1-chloropropane \\
\hline
\end{tabular}




\begin{tabular}{|l|l|}
\hline B56 & 3-(chloromethyl)heptane \\
\hline B57 & 2-chlorooctane \\
\hline B58 & 3-chlorooctane \\
\hline B59 & 4-chlorooctane \\
\hline B60 & 1, 1, 2, 2 - tetrachloroethane \\
\hline B61 & Carbon tetrachloride \\
\hline
\end{tabular}

An attempt has been made to elucidate a QSAR model considering the Number of Carbon Atoms (NCA) and the Number of Chlorine Atoms (NClA). [31],[33],[35]

Table 1.1c lists the experimentally reported $\log \mathrm{EC}_{50}$ values, dipole moment and

$\log \mathrm{EC}_{50}($ vitro $)=14.39 * \mathrm{RE}+0.18 * \mathrm{DP}-0.25 * \mathrm{NCA}-0.91681$

$\mathrm{n}=16$ with $\mathrm{R}^{2}=0.91$ and $\mathrm{S} \mathrm{D}=0.19$

relative energy derived at $\mathrm{B} 3 \mathrm{LYP} / 6-31 \mathrm{G}^{*}$, NCA and the predicted activity. From the table $1.1 \mathrm{c}$, it is clear that the considered descriptors have significant role in predicting the cytotoxicity of the studied chlorinated alkanes and is evidenced by the QSAR equation 1.1

[19],[21],[23

Table 1.1cThe relative energy RE, Dipole Moment DP, number of carbon atom NCA values calculated for compounds classified as set 1 along with their experimental and predicted cytotoxicity values of selected 16 compounds.

\begin{tabular}{|c|c|c|c|c|c|c|}
\hline S. NO & Compound & log EC & RE & DP & NCA & $\begin{array}{c}\text { Predicted log } \\
\mathbf{E C}_{\mathbf{5 0}}\end{array}$ \\
\hline 1 & 1, 3-dichloropropane & 2.99 & 0.27 & 3.61 & 3 & 2.88 \\
\hline 2 & 1, 2-dichloropropane & 3.03 & 0.29 & 2.84 & 3 & 3.06 \\
\hline 3 & $1,1,2$-trichloroethane & 3.04 & 0.29 & 1.31 & 2 & 3.03 \\
\hline 4 & 1, 2-dichlorobutane & 2.61 & 0.28 & 2.75 & 4 & 2.64 \\
\hline 5 & 1-chloro-2, 2-dimethylpropane & 2.30 & 0.28 & 1.96 & 5 & 2.21 \\
\hline 6 & 1-chloro-2-methylbutane & 2.30 & 0.28 & 1.96 & 5 & 2.21 \\
\hline 7 & 1-chloropentane & 2.24 & 0.28 & 2.07 & 5 & 2.26 \\
\hline 8 & 1, 6-dichlorohexane & 2.02 & 0.28 & 3.21 & 6 & 2.16 \\
\hline 9 & 1-chlorohexane & 2.24 & 0.28 & 2.01 & 6 & 1.98 \\
\hline 10 & $1,1-$ dichloro-3, 3 dimethylbutane & 1.67 & 0.28 & 1.93 & 6 & 1.90 \\
\hline 11 & 1-chloroheptane & 1.73 & 0.28 & 2.06 & 6 & 1.98 \\
\hline 12 & 1, 8-dichlorooctane & 1.72 & 0.29 & 3.27 & 8 & 1.85 \\
\hline 13 & 1-chlorooctane & 1.43 & 0.29 & 2.02 & 8 & 1.65 \\
\hline 14 & 1, 9-dichlorononane & 1.78 & 0.29 & 3.84 & 9 & 1.69 \\
\hline 15 & 1-chlorononane & 1.35 & 0.29 & 2.07 & 9 & 1.40 \\
\hline 16 & 1-chlorodecane & 1.44 & 0.29 & 2.02 & 10 & 1.12 \\
\hline
\end{tabular}

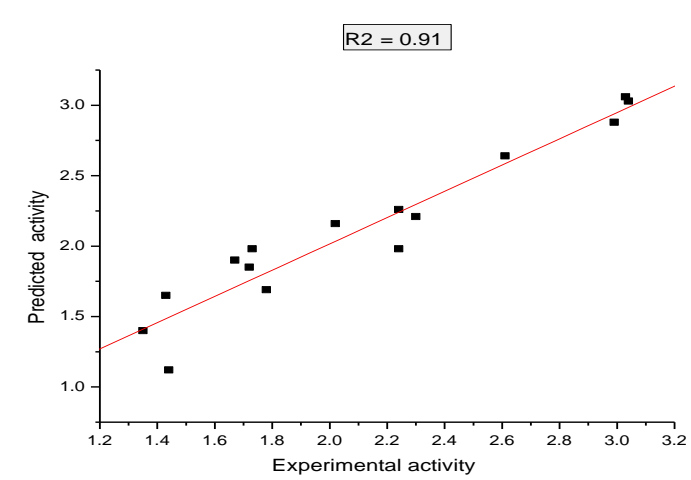

Fig. 1.1a. A plot generated from a linear regression model, using simulated data, with a high value of $\mathrm{R}^{2}$ for the compounds listed in Table 1.1a.

The experimental and predicted cytotoxicity values for the second set of compounds (Table 1.1b) have been predicted using the descriptors such as electrophilicity index, dipole moment and NCA is given in Table 1.1d and the corresponding QSAR equation is given in Equation. 1.2. In this set, presence of more chlorine directly does not give a better QSAR equation than NCA whereas it indirectly 
changes the descriptor (RE) to electrophilicity index $(\omega)$.

$[20],[22],[24]$

\begin{tabular}{|l|l|}
\hline $\log \mathrm{EC}_{50}($ vitro$)=14.58 * \omega+0.05 * \mathrm{DP}-0.199 *$ & $(1.2)$ \\
$\mathrm{NCA}-4.4543$ & \\
$\mathrm{n}=59$ with $\mathrm{R}^{2}=0.70$ and $\mathrm{SD}=0.20$ & \\
\hline
\end{tabular}

Table 1.1d The electrophilicity index $\omega$, Dipole Moment DP, number of carbon atom NCA values calculated for compounds classified as set 2 along with their experimental and predicted cytotoxicity values of selected 59 compounds

\begin{tabular}{|c|c|c|c|c|c|c|}
\hline S.NO & Compound & $\begin{array}{c}\log \\
\text { EC }_{50}\end{array}$ & $\omega$ & DP & NCA & $\begin{array}{c}\text { Predicted } \\
\log \mathrm{EC}_{50}\end{array}$ \\
\hline 1 & 1,2,3-trichloropropane & 2.8 & 0.09 & 3.94 & 3 & 2.69 \\
\hline 2 & 1-chlorobutane & 2.56 & 0.08 & 2.01 & 4 & 2.57 \\
\hline 3 & 1,5-dichloropentane & 2.45 & 0.09 & 3.76 & 5 & 2.38 \\
\hline 4 & pentachloroethane & 2.07 & 0.13 & 1.00 & 2 & 2.27 \\
\hline 5 & 1,1,1,2,2,2,2,3-heptachloropropane & 1.57 & 0.13 & 0.88 & 3 & 1.96 \\
\hline 6 & hexachloroethane & 1.63 & 0.13 & 0.00 & 2 & 2.14 \\
\hline 7 & 1,1-dichloroethane & 2.89 & 0.09 & 2.13 & 2 & 2.84 \\
\hline 8 & $\begin{array}{l}1,3 \text { - dichloro - 2,2-bis (chloromethyl) } \\
\text { propane }\end{array}$ & 2.82 & 0.09 & 0.00 & 5 & 2.12 \\
\hline 9 & trichloromethane & 2.81 & 0.12 & 1.21 & 3 & 2.16 \\
\hline 10 & 1-chloropropane & 2.8 & 0.08 & 2.06 & 3 & 2.75 \\
\hline 11 & 2-chloropropane & 2.8 & 0.08 & 2.16 & 3 & 2.74 \\
\hline 12 & 1,3-dichlorobutane & 2.69 & 0.08 & 3.66 & 4 & 2.62 \\
\hline 13 & 1,4-dichlorobutane & 2.69 & 0.09 & 3.13 & 4 & 2.55 \\
\hline 14 & 1,1-dichloropropane & 2.66 & 0.09 & 2.11 & 3 & 2.68 \\
\hline 15 & 2,2-dichloropropane & 2.66 & 0.09 & 2.33 & 3 & 2.71 \\
\hline 16 & 1,2,3-trichloro-2-methylpropane & 2.62 & 0.09 & 4.18 & 4 & 2.54 \\
\hline 17 & 1,2-dichloro-2-methylpropane & 2.62 & 0.09 & 2.99 & 4 & 2.50 \\
\hline 18 & 1-chloro-2-methylpropane & 2.62 & 0.08 & 2.04 & 4 & 2.53 \\
\hline 19 & 2-chloro-2-methylpropane & 2.62 & 0.08 & 2.17 & 4 & 2.53 \\
\hline 20 & 1,1,1-trichloroethane & 2.58 & 0.11 & 1.89 & 2 & 2.49 \\
\hline 21 & 1,2,3,4-tetrachlorobutane & 2.57 & 0.10 & 0.00 & 4 & 2.26 \\
\hline 22 & 2,3-dichlorobutane & 2.56 & 0.08 & 0.00 & 4 & 2.48 \\
\hline 23 & 2-chlorobutane & 2.56 & 0.08 & 2.10 & 4 & 2.56 \\
\hline 24 & 1,1,3-trichlorobutane & 2.55 & 0.09 & 2.72 & 4 & 2.53 \\
\hline 25 & 1,1,2,3-tetrachloropropane & 2.54 & 0.10 & 1.23 & 3 & 2.44 \\
\hline 26 & 1,2,2,3-tetrachloropropane & 2.54 & 0.11 & 2.22 & 3 & 2.43 \\
\hline 27 & 1,1,2-trichloropropane & 2.53 & 0.09 & 1.40 & 3 & 2.56 \\
\hline 28 & 1,2,2-trichloropropane & 2.53 & 0.09 & 1.63 & 3 & 2.56 \\
\hline 29 & 1,3-dichloro-3-methylbutane & 2.51 & 0.09 & 2.55 & 5 & 2.30 \\
\hline 30 & Tetrachloroethane & 2.51 & 0.10 & 0.00 & 1 & 2.77 \\
\hline 31 & 1,1,1,3-tetrachloropropane & 2.47 & 0.12 & 2.07 & 3 & 2.28 \\
\hline 32 & 1,1-dichlorobutane & 2.42 & 0.09 & 2.03 & 4 & 2.51 \\
\hline 33 & 2,2-dichlorobutane & 2.42 & 0.09 & 2.25 & 4 & 2.48 \\
\hline 34 & 1,1,3,3-tetrachlorobutane & 2.41 & 0.09 & 3.50 & 4 & 2.51 \\
\hline 35 & 1-chloro-3-methylbutane & 2.38 & 0.08 & 1.92 & 5 & 2.35 \\
\hline 36 & 2-chloro-2-methylbutane & 2.38 & 0.08 & 2.12 & 5 & 2.34 \\
\hline 37 & Trichloropropane & 2.34 & 0.09 & 3.94 & 3 & 2.69 \\
\hline 38 & 1,1,1,2-tetrachloroethane & 2.33 & 0.12 & 1.53 & 2 & 2.35 \\
\hline 39 & 2,3-dichloropentane & 2.33 & 0.09 & 2.78 & 5 & 2.31 \\
\hline 40 & 2-chloropentane & 2.32 & 0.08 & 2.04 & 5 & 2.37 \\
\hline 41 & 3-chloropentane & 2.32 & 0.08 & 2.02 & 5 & \\
\hline
\end{tabular}




\begin{tabular}{|c|l|c|c|}
\hline 42 & 1,1,2,2,3-pentachloropropane & 2.27 & 0.11 \\
\hline 43 & $1,1,1,3$-tetrachlorobutane & 2.24 & 0.1 \\
\hline 44 & 1-chloro-3,3-dimethylbutane & 2.2 & 0.09 \\
\hline 45 & $1,1,1$-trichlorobutane & 2.11 & 0.11 \\
\hline 46 & $1,1,1,2$-tetrachloropropane & 2.1 & 0.1 \\
\hline 47 & $1,1,1,3,3$-pentachlorobutane & 2.1 & 0.12 \\
\hline 48 & 2-chlorohexane & 2.09 & 0.08 \\
\hline 49 & 3-chlorohexane & 2.09 & 0.08 \\
\hline 50 & 2-chloro-2,3,3-trimethylbutane & 2.03 & 0.08 \\
\hline 51 & 2,5-dichloro-2,5-dimethylhexane & 1.86 & 0.09 \\
\hline 52 & 2-chloroheptane & 1.85 & 0.08 \\
\hline 53 & 3-chloroheptane & 1.85 & 0.08 \\
\hline 54 & 1,1,1,3-tetrachloro-4-methylpentane & 1.82 & 0.11 \\
\hline 55 & 1-chloro-2,2,4-trimethylpentane & 1.79 & 0.08 \\
\hline 56 & 3-(chloromethyl)heptane & 1.67 & 0.08 \\
\hline 57 & 2-chlorooctane & 1.62 & 0.08 \\
\hline 58 & 3-chlorooctane & 1.62 & 0.08 \\
\hline 59 & 4-chlorooctane & 1.62 & 0.08 \\
\hline & & & $2)$
\end{tabular}

2) Peer Mohamed M., Jayaprakash P., Nageshwari M., Rathika Thaya Kumari C., Sangeetha P., Sudha S., Mani G., Lydia Caroline M., Crystal growth, structural, spectral, thermal, linear and nonlinear optical characterization of a new organic nonlinear chiral compound:

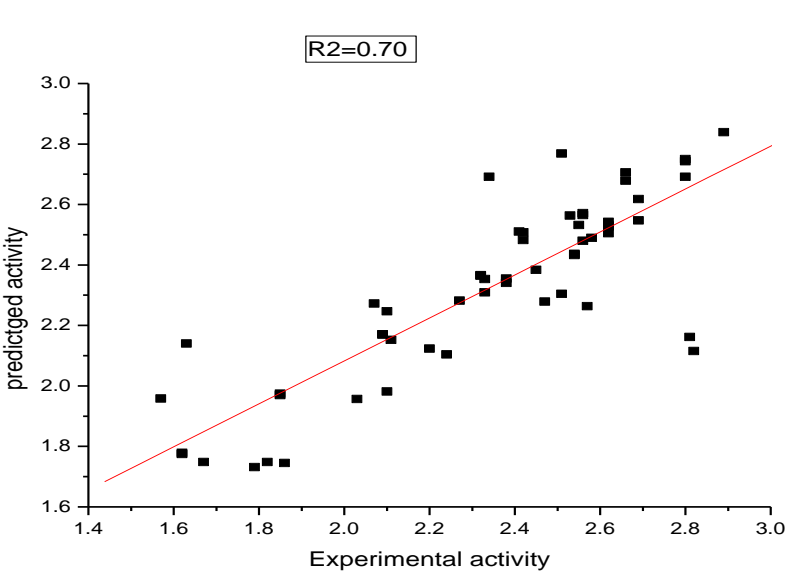

3) L-tryptophan-fumaric acid-water (1/1/1) suitable for laser frequency conversion, 2017, Journal of Molecular Structure, V-1141, PP-551-562

4) Jayaprakash P., Sangeetha P., Kumari C.R.T., Caroline M.L., Investigation on the growth, spectral, lifetime, mechanical analysis and third-order nonlinear optical studies of L-methionine admixtured D-mandelic acid single crystal: A promising material for nonlinear optical applications, 2017, Physica B: Condensed Matter, V-518, PP-1-12

5) Nageshwari M., Kumari C.R.T., Vinitha G., Mohamed M.P., Sudha S., Caroline M.L., Crystal growth, structural, spectral, thermal, dielectric, linear and nonlinear optical characteristics of a new organic acentric material: L-Methionine-Succinic acid (2/1), 2018, Journal of Molecular Structure, V-1155, PP-101-109

6) Jayaprakash P., Sangeetha P., Kumari C.R.T., Baskaran I., Caroline M.L., Growth and characterization of 1-asparagine monohydrate admixtured dl-mandelic acid nonlinear optical single crystal, 2017, Journal of Materials Science: Materials in Electronics, V-28, PP-18787-18794

7) Rathika Thaya Kumari C., Nageshwari M., Jayaprakash P., Sangeetha P., Sudha S., Lydia Caroline M., Investigation on growth, optical, thermal, mechanical, dielectric, LDT studies of sulphanilic acid monohydrate: A promising third-order nonlinear optical material, 2017, Journal of Nonlinear Optical Physics and Materials, V-26, I - 2,

Figure 1.1b Plot illustrating the experimental cytotoxicity against the predicted values for the compounds listed in Table 3.1b with a high value of $R^{2}$.

\section{CONCLUSION}

From the QSAR analysis of the chlorinated alkanes, [26],[28],[30]it is clear that the compounds having a greater number of $\mathrm{C}$-atoms gives better prediction with relative energy than the electrophilicity index, whereas, for the more chlorinated compounds, the electrophilicity index influences the QSAR model. [25],[27],[29]

\section{REFERENCES}

1). Nageshwari M., Jayaprakash P., Kumari C.R.T., Vinitha G., Caroline M.L., Growth, spectral, linear and nonlinear optical characteristics of an efficient semiorganic acentric crystal: L-valinium L-valine chloride, 2017, Physica B: Condensed Matter, V-511, PP -1-9,Sangeetha P., Jayaprakash P., Nageshwari M., Rathika Thaya Kumari C., Sudha S., Prakash M., Vinitha G., Lydia Caroline M., Growth and characterization of an efficient new NLO single crystal L-phenylalanine D-methionine for frequency conversion and optoelectronic applications, 2017, Physica B: Condensed Matter, V-525, PP-164-174

8) Thaya Kumari C.R., Nageshwari M., Raman R.G., Caroline M.L., Crystal growth, spectroscopic, DFT computational and third harmonic generation studies of nicotinic acid, 2018, Journal of Molecular Structure, V-1163, PP-137-146

9) Nageshwari M., Kumari C.R.T., Sangeetha P., Vinitha G., Caroline M.L., Third order nonlinear optical, spectral, dielectric, laser damage threshold, and photo luminescence characteristics of an efficacious semiorganic acentric crystal: L-Ornithine monohydrochloride, 2018 , Chinese Journal of Physics, V-56, I - 2, PP-502-519

10) Kumari C.R.T., Jayaprakash P., Nageshwari M., Mohamed M.P. Sangeetha P., Caroline M.L., Growth, optical, photoluminescence, dielectric, second and third order nonlinear optical studies of benzoyl valine acentric crystal, 2017, Molecular Crystals and Liquid Crystals, V-658,I - 1, PP-186-197

11) Rathika Thaya Kumari C., Nageshwari M., Sudha S., Lydia Caroline M., Mani G., Influence of uranyl on the growth, linear, laser damage threshold and nonlinear optical studies on potential nonlinear optical single crystal: L-Alanine, 2015, Journal of Chemical and Pharmaceutical Sciences, V-2015-October,PP-166-170,

12) Rathika Thaya Kumari C., The detailed study ontwo dimensional hydrogen atom, 2013, Middle - East Journal of Scientific Research, V-17,I-12, PP-1900-1902

13) Rathika Thaya Kumari C., Theoretical study about the measurement of ultrasonic velocity using different methods, 2013, Middle - East Journal of Scientific Research, V-17, PP-12-

14) Mukunthan A., Rathika Thaya Kumari C., Nageshwari M., Prakash D., The effect of super saturation and crystal growth 
kinetics of L-arginine per chlorate, 2013, Middle - East Journal of Scientific Research, V-17,I-12 PP-1825-1827

15) Sree Latha R., Vijayaraj R., Azhagiya Singam E.R., Chitra K., Subramanian V., 3D-QSAR and Docking Studies on the HEPT Derivatives of HIV-1 Reverse Transcriptase, 2011, Chemical Biology and Drug Design, V-78,I-12, PP-1816-1819

16) Sree Latha R., Vijayaraj R., Padmanabhan J., Azhagiya Singam E.R. Chitra K., Subramanian V., 3D-QSAR studies on the biological activity of juvenile hormone mimetic compounds for Culex pipiens Larvae, 2013, Medicinal Chemistry Research, V-22, I-12,PP-418-426

17) Subashini G.,A simple proof on coloring of dominated special graphs, Indian Journal of Science and Technology,V-7,I-,PP-5-6,Y-2014

18) Ramya N.,On coloring of corona graphs, Indian Journal of Science and Technology,V-7,I-SUPPL2,PP-9-11,Y-2014.

19) Ramya N., On colourings of wheel graph (Wn), Indian Journal of Science and Technology,V-7,I-,PP-72-73,Y-2014.

20) Ramya N., On star chromatic number of $P 3$ (n), Indian Journal of Science and Technology,V-7,I-,PP-7-8,Y-2014.

21) Vijayaragavan S.P.,An investigative expert that's general FBG sensors,International Journal of Mechanical Engineering and Technology,V-8,I-8,PP-1500-1505,Y-2017

22) Vijayaragavan S.P.,Equalization routing protocol for Wi-Fi sensor strategy,International Journal of Mechanical Engineering and Technology,V-8,I-8,PP-1662-1666,Y-2017

23) Karthik B., Kiran Kumar T.V.U., Vijayaragavan P., Bharath Kumaran E.,Design of a digital PLL using 0.35 $\hat{\mathrm{I}}^{1 / 4 \mathrm{~m}}$ CMOS technology,Middle East Journal of Scientific Research,V-18,I-12,PP-1803-1806,Y-2013

24) Kanniga E., Selvaramarathnam K., Sundararajan M.,Kandigital bike operating system,Middle - East Journal of Scientific Research,V

25) Jasmin M., Vigneshwaran T., Beulah Hemalatha S.,Design of powe aware on chip embedded memory based FSM encoding in FPGA,International Journal of Applied Engineering Research,V-10,I-2,PP-4487-4496,Y-2015

26) Jasmin M.,Optimization techniques for low power VLSI circuits,Middle - East Journal of Scientific Research,V-20,I-9,PP-1082-1087,Y-2014

27) Jasmin M., Vigneswaran T.,Fuzzy controller for error control of on Chip communication,2017 International Conference on Algorithms, Methodology, Models and Applications in Emerging Technologies, ICAMMAET 2017,V-2017-January,I-,PP-1-5,Y-2017

28) Ramya N., On star chromatic number of $P 3$ (n),Indian Journal of Science and Technology,V-7,I-,PP-7-8,Y-2014.

29) Cauchy Multiplication of Euler Summable series in Ultrametric Fields, PNNVS R.Deepa Commentationes Mathematicae 53 ((1)), 73- 79

30) Kumaravel A., Meetei O.N.,An application of non-uniform cellular automata for efficient cryptography,Indian Journal of Science and Technology,V-6,I-SUPPL5,PP-4560-4566,Y-2013

31) Kumaravel A., Meetei O.N.,An application of non-uniform cellular automata for efficient cryptography,2013 IEEE Conference on Information and Communication Technologies, ICT 2013,V-,I-,PP-1200-1205,Y-2013

32) Kumarave A., Rangarajan K.,Routing alogrithm over semi-regular tessellations,2013 IEEE Conference on Information and Communication Technologies, ICT 2013,V-,I-,PP-1180-1184,Y-2013

33) Gopinath S., Sundararaj M., Elangovan S., Rathakrishnan E.,Mixing characteristics of elliptical and rectangular subsonic jets with swirling co-flow,International Journal of Turbo and Jet Engines, V-32,I-1,PP-73-83,Y-2015

34) Rathika Thaya Kumari C., A brief review on the impacts of tropospheric ozone on biological systems, 2013, Middle - East Journal of Scientific Research, V-17,I-12, PP-1820-1824

35) Mukunthan A., Rathika Thaya Kumari C., Nageshwari M., Prakash D., A brief review on the applications of fluorescent spectroscopy in biomedical diagnosis, 2013, Middle - East Journal of Scientific Research, V-17,I-12, PP-1828-183

\section{AUTHORS PROFILE}

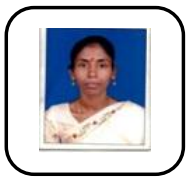

R.Suganya Assistant Professor, Department of Physics Bharath Institute of Higher Education and Research, Chennai, India

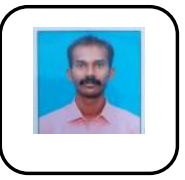

Dr.S.Bharanidharan Associate Professor, Department of Physics, Bharath Institute of Higher Education and Research, Chennai, India

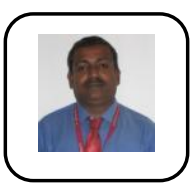

Dr.R.Velavan Associate Professor, Department of Physics, Bharath Institute of Higher Education and Research, Chennai, India 